LIGANG WU, Ph.D. ${ }^{1}$

(Corresponding author)

E-mail: ligangwu@yeah.net

ZIBAO LU, Ph.D. 2,3

E-mail: luzibao@163.com

GE GUO, Ph.D. 4

E-mail: geguo@yeah.net

${ }^{1}$ Department of Electrical Engineering

Shanxi Datong University, Datong, Shanxi 037003, China

2 School of Control Science and Engineering

Dalian University of Technology, Dalian 116023, China

${ }^{3}$ College of Physics and Electronic Information

Anhui Normal University, Wuhu 241000, China

${ }^{4}$ School of Information Science and Technology

Dalian Maritime University, Dalian 116026, China
Information and Communication Technology

Preliminary Communication

Submitted: 12 Oct. 2015

Accepted: 21 Sep. 2016

\title{
ANALYSIS, SYNTHESIS AND EXPERIMENTS OF NETWORKED PLATOONS WITH COMMUNICATION CONSTRAINTS
}

\begin{abstract}
This paper investigates the analysis and synthesis of networked vehicle platoons with communication delays, packet dropouts and disorders. In order to deal with the effects of the communication constraints, we introduce a novel Smart Data Processor (SDP) for each vehicle, by which the latest data packets from logic Data Packet Processor and the matched data packet from its Buffer can be obtained. Based on this mechanism, a leader-predecessor-follower control strategy is proposed. In order to guarantee the as ymptotic and string stability, the platoon control problem is transformed into a multi-objective Ho-type synthesis problem with the multiple time-varying delays. A sufficient condition for designing the controller gain is derived by solving a set of linear matrix inequalities. Numerous simulations and experiments with laboratory scale Arduino cars show the efficiency of the proposed methods.
\end{abstract}

\section{KEY WORDS}

platoon; communication constraints; Smart Data Processor; Ho-type performance;

\section{INTRODUCTION}

The past several decades have witnessed a considerable increase of highway traffic, so the traffic congestion, air pollution, enormous economic loss and even numerous casualties have become a series of serious problems in many metropolises, especially in China. Obviously, a feasible solution for these problems is to increase road capacity by maintaining a small inter-vehicle distance. As this would be unsafe for human drivers, autonomous vehicular cooperative control will be required. The platoons of autonomous vehicles have attracted extensive interests in intelligent vehicle highway systems (IVHS) or automated highway/vehicle systems (AHVS) due to its potential to largely benefit the road traffic [1-3]. Up to now, control of platoon of vehicles has been researched in different aspects and from different viewpoints [4]. To name just a few, Barooah [5] introduced a mistuning-based control method to improve the stability margin of vehicular platoon; Dunbar and Derek [6] designed a distributed receding horizon controller and derived sufficient conditions to ensure string stability; Kianfar [7] performed platoon control in the real world, among many others.

Since vehicles in a platoon are dynamically coupled by feedback control laws, acting on one vehicle may inevitably affect others or even amplify as they propagate upstream along the platoon [8]. Hence, the problem of string stability or stability of the platoon as a whole is an important aspect of vehicle platoon control, which is beyond stabilizing each of the individual vehicles. However, it is not stringently required in the case of variable spacing policy without inter-vehicle communication [9]. Instead, we are interested in the case of platoon control with constant spacing strategy.

What are the main underlying causes and remedies for string instability problem? Generally speaking, the disturbances in the system are the main factor. The lead vehicle acceleration/deceleration is the primary disturbance existing in the platoon control system, but the inter-vehicle communication involved in vehicle platoon control can often introduce intermediate uncertainties (such as transmission delays, packet dropouts and disorders), which may yield string instability problem or large steady state errors. In recent years, network communication has developed rapidly. Information exchange based on network has become increasingly popular. Emerging technologies in Vehicle to Vehicle (V2V) $[10,11]$ and/or Vehicle to Infrastructure (V2I) wireless communication [12] can drive a platoon control system that is more efficient, more robust 
and less expensive, but also it is almost inevitably accompanied by the above three phenomena that may yield string instability. Compared with the traditional network communication systems, the control of the vehicular ad hoc network has several challenges. For instance, VANETs have the short connection time and quick variations of the wireless channel in the wireless vehicular networks due to fast moving vehicles.

Up to date, there have been a few research results on control of vehicle platoons via communication networks in the literature. Zhang $[13,14]$ studied the influence of information flow topologies on closed-loop stability of vehicle platoon with rigid formation and improved the vehicular platoon with undirected topology and asymmetric control by stability margin [15]. To mention them, the effect of the communication delay on string stability in a platoon has been investigated in [16-18]. Guo [19, 20] considered the network communication with time-varying delays in the platoon control and structured the Lyapunov framework or the guaranteed cost $\mathrm{H} \infty$ controller. Ghasemi [21] introduced the stability and string stability of a predecessor-following platoon of vehicles with two time delays, i.e., one in the inter-vehicle distance and the other in the relative velocity information channels. Hakan [22] formulated the homogenous platoon operating under the leader and predecessor following scheme with communication constraints as a multi-objective Ho-type synthesis problem. Oncu [23] designed Cooperative Adaptive Cruise Control (CACC) based on network perspective and also presented a network modelling framework in the platoon system, but the issue of how to design the controller and handle the effect of time-varying communication delay was not resolved.

In general, the results obtained for platoon control systems are still very limited in at least the following two aspects: (1) The existing results are most concerned with the case in which the information available to a vehicle is homogenous, whereas little attention has been paid to the problem of control with 'heterogeneous information feedback', such as partially supplied by an on-board device and partially via a wireless communication channel. (2) A majority of results on platoon control with inter-vehicle communication only consider one aspect of the communication constraints, whereas the synthesis problems with simultaneous consideration of the above important communication issues mentioned previously has been hardly addressed.

At a deeper level, although the platoon control system with communication constraints has been studied, it still leaves some room for further improvement. To the best of our knowledge, the platoon control problem, which considers the synthesis of the time-varying delays, disorders, dropout and asynchronous data packets, has been investigated.
The aim of this paper is to address the platoon control problem with communication delays, disorders, dropout and asynchronous data packets between vehicles by structuring a novel Smart Data Processor (SDP). Under this mechanism each vehicle in the platoon control system can successfully select the latest data packets of the leader vehicle by using a Logic Data Packet Processor and the matched ones of its own from its Buffer. Based on these data packets, a new state-feedback control law for homogenous platoons is given. Then, the platoon control problem is transformed into a multi-objective Ho-type synthesis problem, which can guarantee asymptotic and string stability of the platoon and the desired level of robustness against the communication constraints. The contributions of this paper are summarized in the following aspects:

1) Compared with [19] and [24], this paper further considers the more complicated communication constraints involving communication delays, packet dropouts and disorders. It implies that the methods used in [19] and [24] may be no longer valid, especially when the data packet disorders happens. To deal with this problem, we have developed a new control strategy by introducing a novel Smart Data Processor (SDP). Based on this strategy, we have established a discrete-time closed loop system with communication constraints.

2) Unlike the mechanism of [25] and [26], a novel SDP mechanism is proposed to deal with the asynchronous data packets arising from communication constraints. By the use of Buffer and Event Trigger, the matched data packets from both the vehicle and the leader can be found for control update.

3) We have formulated asymptotical stability and string stability of the platoon control system as a multi-objective Ho-type synthesis problem: By employing Lyapunov method, a sufficient condition for controller design is obtained.

The rest of this paper is organized as follows. Section 2 presents the vehicle dynamics discrete-time model and the problem formulation. In Section 3, Hotype performance objectives are given. Section 4 presents the simulation and experiments with laboratory scale Arduino cars, showing the effectiveness of the proposed methods. Section 5 summarizes the main conclusions.

\section{PROBLEM FORMULATION}

\subsection{Vehicle following objective}

Consider $n+1$ vehicles running on a horizontal road as depicted in Figure 1, describing a typical homogeneous platoon of vehicles equipped with the wireless communication functionality and on-board sensors 
(e.g. using radar). In many practical cases, a platoon may be composed of a group of vehicles with the same type or similar dynamics, e.g. trucks or passenger cars [14]. Thus, it is reasonable to consider the homogeneous platoon in our study. The main objective of each vehicle is to drive two adjacent vehicles to maintain a desired distance $e_{d}^{p}$. Define the spacing error between two consecutive vehicles as

$e_{i}^{p}(k)=q_{i-1}(k)-q_{i}(k)-L-e_{d}^{p}, \quad 1 \leq i \leq n$

where $q_{i}$ is the $i$-th vehicle's position and $L$ denotes the length of vehicle. In other words, the vehicle following control objective is that the spacing errors $e_{l}^{p}(k)$ should converge to zero.

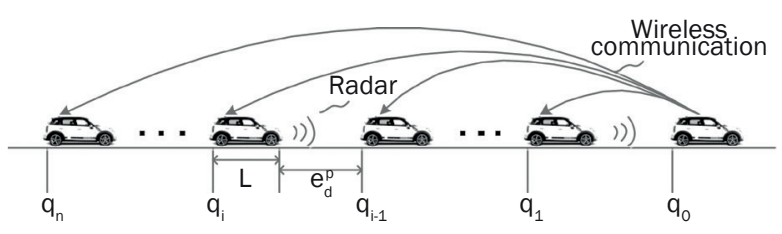

Figure 1 - Wireless communication-equipped homogeneous autonomous vehicle platoon

\subsection{Vehicle dynamics model and networked platoon configuration}

Various models for vehicle dynamics have been used in the platoon system. Here, the vehicular discretized models given in [24] are used,

$$
\left\{\begin{aligned}
q_{i}(k+1)= & q_{i}(k)+T v_{i}(k)+\left(\eta^{2} e^{-T / \eta}+\eta T-\eta^{2}\right) a_{i}(k) \\
& +\left[\eta^{2}\left(e^{-T / \eta}+1\right)+T(-\eta+T)\right] u_{i}(k) \\
v_{i}(k+1)= & v_{i}(k)+\left(-\eta e^{-T / \eta}+\eta\right) a_{i}(k)+ \\
& +\left[\eta\left(e^{-T / \eta}-1\right)+T\right] u_{i}(k) \\
a_{i}(k+1)= & e^{-T / \eta} a_{i}(k)+\left(-e^{-T / \eta}+1\right) u_{i}(k)
\end{aligned}\right.
$$

where $v_{i}$ and $a_{i}$ denote the $i$-th vehicle's velocity and acceleration, respectively; $u_{i}$ is the external input signal to be designed; $T$ is the sampling interval; $\eta$ is the engine time constant. For the dynamics of the leader vehicle, we allow that it is different from other vehicles only in the control law $u_{0}(k)$.

It is well-known that the key technology of platoon is wireless communication of conveying the information about being chain vehicles so that every vehicle could receive information from some other vehicles. Generally speaking, there are the following three types of communication: predecessor-follower strategy, leader-predecessor-follower strategy, and communication of all vehicles. This paper adopts leader-predecessor-follower, i.e. the vehicles not only receive the information of the leader vehicle, but they also receive the information of its directly preceding vehicle. Here, the former data are obtained by wireless network, but the latter are passed through the on-board sensors.

This paper focuses on designing an appropriate control law $u_{i}$ for any $1 \leq i \leq n$ such as longitudinal control of discrete-time vehicle platoon (Expression 2) shown in Figure 1 that can be asymptotically achieved with a desired distance between vehicle $i$ and $i-1$ and string stability guaranteed.

Notice that the state information of leader vehicle such as its position, velocity and acceleration can be only transmitted to vehicle $i$ via a wireless communication network. The communication networks are usually unreliable, so the communication delay, packet dropout and disorder may occur during the data packet transmission. It seriously affects or even destroys the stability of the platoon system. In order to solve the above problem, inspired by the work in [25] and [26], we have structured a novel data packet processing mechanism for each vehicle $i$, whose configuration is shown in Figure 2. The key point of this mechanism is how to effectively perform the combined processing in selecting the latest data packet of leader vehicle and its matched data packets of vehicle $i$. This mechanism is explained in detail as follows.

To be specific, firstly, every vehicle $i$ makes its state packed as a data packet $\sigma_{i}(k)$ with the time stamp $k$ by using the data packet generator (DPG), where $\sigma_{i}(k)^{T}=\left[q_{i}(k), v_{i}(k), a_{i}(k)\right], \quad i=1,2, \ldots, n$ and $\sigma_{0}(k)$ is the leader vehicle information. At the time instant $k$, the leader vehicle needs to send $\sigma_{0}(k)$ to the following vehicle $i$ through a wireless network. On account of the existence of transmission delay and packet dropout, vehicle $i$ does not immediately receive the exact information of data packet $\sigma_{0}(k)$.

Moreover, since the data packets at different time instant $k$ may experience different delays for other following vehicles, it may lead to the packet disorder. Therefore, a Smart Data Processor (SDP), which consists of a Logic Data Packet Processor (LDPP), a Buffer and an Event Trigger (ET), is introduced to surmount the combined effect of time-varying delay, packet dropout and disorder by successfully completing two tasks: (1) selecting the latest data packet from the leader vehicle; (2) picking the data packet of vehicle $i$ matched with the latest one from the leader vehicle. The LDPP is composed of a comparator and a storer, where the comparator is employed to logically compare the time stamp of the newly arrived packet with the one already in LDPP to select the newest data packet, and the storer is used to keep the newest data packet after comparison. Hence, the LDPP can effectively solve the problem of packet disorder. Denote by $h_{i}^{k}$ the corresponding time of the latest data packet from the leader vehicle to vehicle $i$ at time instant $k$. So, the mechanism of LDPP can be described as follows:

Given $\sigma_{0}(0)$, let $h_{i}^{0}=0$ and $k=0$. 


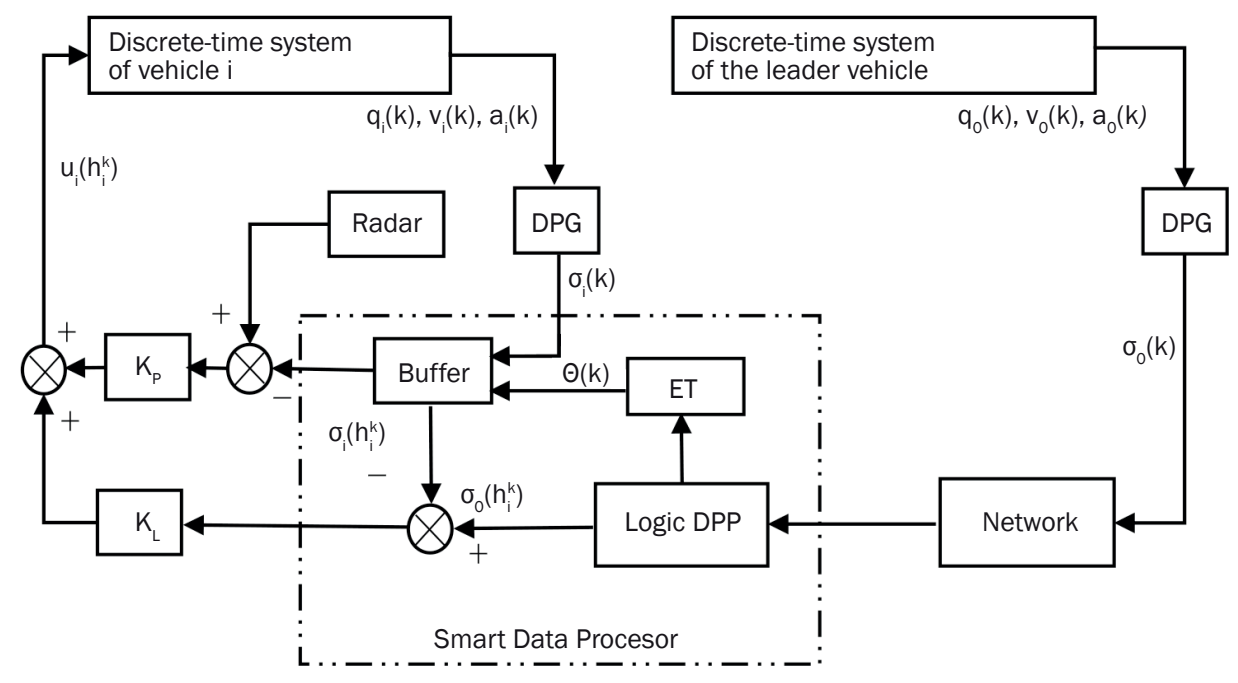

Figure 2 - Data processing mechanism of vehicle $i$

Step 1: At the time instant $k$, the LDPP changes its output to $\sigma_{0}(k)=\sigma_{0}\left(h_{i}^{k}\right)$. Let $h_{i}^{k+1}=h_{i}^{k}$.

Step 2: If a packet $\sigma_{0}(j)$ arrives at the time instant $k+1$ and $j>h_{i}^{k+1}$, LDPP stores $\sigma_{0}(j)$. Let $h_{i}^{k+1}=j$.

Step 3: Repeat Step 2 until no data packets arrive at the instant $k+1$. Let $k=k+1$ and go to Step 1 .

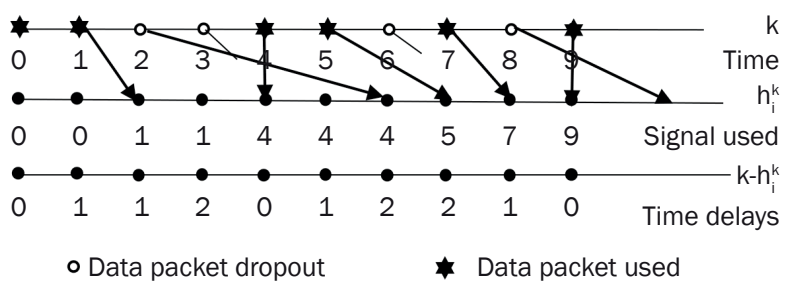

Figure 3 - An example to explain how the Logic DPP works

Remark 1: Figure 3 gives a detailed example for the data processing in the Logic DPP. Form Figure 3 , it is obvious that $h_{i}^{k} \leq \mathrm{k}$ and $h_{i}^{k} \leq h_{i}^{k+1} \leq k+1$ are found. Once one data packet is disordered, LDPP must drop it out; for example, the second data packet in Figure 3 is dropped out by LDPP because it is disordered with the fourth data packet when it arrives at LDPP.

Because of the effect of the communication delay, the data packets of the leader vehicle at time instant $k$ may arrive at the controller of vehicle $i$ at a different time, which means that asynchronous data packets will be used for the computation of controller of vehicle $i$. Unfortunately, it is undesirable for control design and analysis. Thus, to avoid the asynchronous problem of the sequence time, the buffer and the event trigger (ET) are introduced in response to LDPP. The buffer is to store the state information sequence $M$ of vehicle $i$ from $h_{i}^{k}$ to $k$ time, where $M=\left[\sigma_{i}\left(h_{i}^{k}\right), \sigma_{i}\left(h_{i}^{k}+1\right), \ldots, \sigma_{i}(k)\right]$. The ET can generate a trigger signal if the output of the LDPP is updated. The trigger signal for each vehicle $i$ is set as a two-valued variable $\theta_{i}(k)$, which can be modelled as: $\theta_{i}(k)=\left\{\begin{array}{l}1, \text { the output of the LDPP of vehicle } i \\ \text { is updated } \\ 0, \text { otherwise }\end{array}\right.$

If the ET output $\theta_{i}(k)=0$, then the newest data in the Logic DPP is received by vehicle $i$ at the last instant. Otherwise, once the trigger signal $\theta_{i}(k)=1$, the buffer will be authorized to select the corresponding data packet $\sigma_{i}\left(h_{i}^{k}\right)$ from the sequence $M$ in consistency with the received latest data packet $\sigma_{0}\left(h_{i}^{k}\right)$ of the leader vehicle. After that, the sequence $M$ is updated accordingly. The mechanism of the buffer can be described as follows:

Give $\theta_{i}(k)=0$, let $h_{i}^{0}=0$ and $k=0$.

Step 1: At the time instant $k$, the buffer changes its output to $\sigma_{i}(k)=\sigma_{i}\left(h_{i}^{k}\right)$. Let $h_{i}^{k+1}=h_{i}^{k}$.

Step 2: At the time instant $k+1$, if $\theta_{i}(k)=1$, the buffer output the data $\sigma_{0}(j)$, let $h_{i}^{k+1}=j$ and update the state information sequence in the buffer as $M=\left[\sigma_{i}(j), \sigma_{i}(j+1), \ldots, \sigma_{i}(k+1)\right]$. Otherwise, the buffer maintains the former output data $\sigma_{i}\left(h_{i}^{k+1}\right)$.

Step 3: Let $k=k+1$ and go to Step 1.

Therefore, both data packets $\sigma_{i}\left(h_{i}^{k}\right)$ and $\sigma_{0}\left(h_{i}^{k}\right)$ are sent to the controller of vehicle $i$ for computation. Denote the velocity and acceleration errors by $e_{i}^{v}(k)=v_{i-1}(k)-v_{i}(k)$ and $e_{i}^{a}(k)=a_{i-1}(k)-a_{j}(k)$, respectively. Based on the state error information, let $e_{i}(k)=\left[e_{i}^{p}(k), e_{i}^{v}(k), e_{i}^{a}(k)\right]^{T}$, we propose the following control law for vehicle $i(i \geq 2)$

$$
\begin{aligned}
u_{i}(k)= & K_{P} e_{i}(k)+K_{L}^{p}\left(q_{0}\left(h_{i}^{k}\right)-q_{i}\left(h_{i}^{k}\right)-i L-i e_{d}^{p}\right) \\
& +K_{L}^{v}\left(v_{0}\left(h_{i}^{k}\right)-v_{i}\left(h_{i}^{k}\right)\right)+K_{L}^{a}\left(a_{0}\left(h_{i}^{k}\right)-a_{i}\left(h_{i}^{k}\right)\right)
\end{aligned}
$$

where $K_{P}=\left[K_{P}^{P}, K_{P}^{v}, K_{P}^{a}\right], K^{p}, K_{L}^{v}$ and $K_{L}^{a}$ are the controller gains to be designed later.

Let $\tau_{i}^{k} \triangleq k-h_{i}^{k}$, so $\tau_{i}^{k}$ can be regarded as a network transmission delay of the data packet of the leader vehicle at instant $k$ received by vehicle $i$ via wireless communication network. Note that the effects of the 
network-induced delays and packet losses can be synchronously represented by $\tau_{i}^{k}$ and can be distinguished according to different values of $\tau_{i}^{k}$. From the definition of $\tau_{i}^{k}$, it is easily known that $\tau_{i}^{k+1} \leq \tau_{i}^{k}+1$, then we need to analyze the following two cases:

Case 1: If $\tau_{i}^{k+1}=\tau_{i}^{k+1}$, it indicates that no new data packet has arrived at LDPP at instant $k+1$. The output of LDPP is invariable, so delay $\tau_{i}^{k}$ of the leader vehicle is increased by one.

Case 2: If $\tau_{i}^{k+1} \leq \tau_{i}^{k}$, it indicates that a new data packet with the delay $\tau_{i}^{k+1}$ is accepted by LDPP up to time $k+1$ and this packet will be used at time $k+1$. Let $N_{i}^{k}=\tau_{i}^{k+1}$ $\tau_{i}^{k}$, then $N_{i}^{k}$ is the number of consecutive packet losses from the leader vehicle to vehicle $i$.

In order to maintain a certain communication performance, the following assumptions are made: Assumption 1: $\tau_{i}^{k}$ is upper bounded by $\tau_{\max }$, i.e. $0 \leq \tau_{i}^{k} \leq \tau_{\text {max }}$

Assumption 2: The number of consecutive data dropouts $N_{i}^{k}$ is upper bounded by $N_{d}$.

Thus, the control law in Expression 3 for vehicle $i$ can be rewritten as

$$
\begin{aligned}
u_{i}(k)= & K_{p} e_{i}(k)++K_{L}^{p}\left(q_{0}\left(k-\tau_{i}^{k}\right)-q_{i}\left(k-\tau_{i}^{k}\right)-i L-i e_{d}^{p}\right) \\
& +K_{L}^{v}\left(v_{0}\left(k-\tau_{i}^{k}\right)-v_{i}\left(k-\tau_{i}^{k}\right)\right)+ \\
& +K_{L}^{a}\left(a_{0}\left(k-\tau_{i}^{k}\right)-a_{i}\left(k-\tau_{i}^{k}\right)\right)
\end{aligned}
$$

As the leader vehicle is the directly preceding vehicle for the first vehicle in the platoon, the first vehicle is run without the communication effect. The control law for the first vehicle is given in the following form

$u_{i}(k)=\left(K_{P}+K_{L}\right) e_{1}(k)$

In order to evaluate the performance of the first vehicle, we define the following signal

$$
\xi_{i}(k)=F_{1} e_{1}(k)+G_{1}\left(u_{1}(k)-u_{0}(k)\right)
$$

In the above expression, we can pick the error signals of interest by $F_{1}$, such as the spacing, velocity and acceleration error. However, $G_{1}$ is capable of adjusting the contribution of the control inputs $u_{1}$ and $u_{0}$ to minimize the cost. Corresponding to the first vehicle, the performance output signal for other following vehicles is defined

$$
\xi_{i}(k)=F e_{i}(k)+G\left(u_{i}(k)-u_{i-1}(k)\right)
$$

By means of the control law in Expression 4, the change in the control input from (i-1)-th to $i$-th vehicle is written as

$$
\begin{aligned}
u_{i}(k)-u_{i-1}(k)= & K_{P} e_{i}(k)-K_{P} e_{i-1}(k)+ \\
& +K_{L} e_{i}(k)+K_{L} \Theta_{i}(k)
\end{aligned}
$$

where $\Theta_{i}(k)$ is a disturbance variable for the simplicity of our notation.

Remark 2: Due $q_{i}\left(k-\tau_{i}^{k}\right)=q_{i}(k)+\Delta_{i}(k), q_{i-1}\left(k-\tau_{i-1}^{k}\right)=q_{i-1}(k)+\Delta_{i-1}(k)$ and $q_{0}\left(k-\tau_{i}^{k}\right)-q_{0}\left(k-\tau_{i-1}^{k}\right)=\Phi_{i}(k), \Theta_{i}(k) \triangleq \Delta_{i}(k)+\Delta_{i-1}(k)+\Phi_{i}(k)$ is reasonable.
In other words, Expression 8 can be used for the next analysis.

The closed-loop form of vehicle $i$ can be represented by

$$
\begin{aligned}
e_{i}(k+1) & =A e_{i}(k)+B\left(u_{i}(k)-u_{i-1}(k)\right) \\
\text { where } A & =\left[\begin{array}{ccc}
1 & T & \eta^{2} e^{-T / \eta}+\eta T-\eta^{2} \\
0 & 1 & -\eta e^{-T / \eta}+\eta \\
0 & 0 & e^{-T / \eta}
\end{array}\right], \\
B & =\left[\begin{array}{c}
\eta^{2}\left(e^{-T / \eta}+1\right)+T(-\eta+T) \\
\eta\left(e^{-T / \eta}-1\right)+T \\
-e^{-T / \eta}+1
\end{array}\right]
\end{aligned}
$$

Substituting Expression 8 into Expression 9, for vehicle $i \geq 2$, the closed-loop form can be written as

$$
\begin{aligned}
e_{i}(k+1)= & \left(A+B K_{P}+B K_{L}\right) e_{i}(k)- \\
& -B K_{P} e_{i-1}(k)+B K_{L} \Theta_{i}(k) \\
\xi_{i}(k)=( & \left.F+G K_{P}+G K_{L}\right) e_{i}(k)- \\
& -G K_{P} e_{i-1}(k)+G K_{L} \Theta_{i}(k)
\end{aligned}
$$

Similarly, the closed-loop form for the first vehicle can be described as

$$
\begin{aligned}
& e_{1}(k+1)=\left(A+B K_{P}+B K_{L}\right) e_{1}(k)-B u_{0}(k) \\
& \xi_{1}(k)=\left(F_{1}+G_{1} K_{P}+G_{1} K_{L}\right) e_{1}(k)-G_{1} u_{0}(k)
\end{aligned}
$$

Therefore, the following objectives of each following vehicle need to be accomplished:

1) The longitudinal control problem of the platoon system to be addressed in this paper can be formulated as the stabilization problem of the discrete-time error system (Expressions 10 and 12) with multiple delays.

2) As an additional requirement, the global performance of the platoon system is string stable. Although the spacing error $e_{i}^{p}(k)$ may guarantee convergence to zero in steady state, it gives no specific hints or restrictions on the spacing error in transient state for all the time.

Next, the main results of this paper for solving these problems are given.

\section{3. $\mathrm{H}_{\infty}$-TYPE PERFORMANCE OBJECTIVES}

In this section, the novel $\mathrm{H}_{\infty}$-type performance objectives are proposed to guarantee asymptotic and string stability of the platoon system. By solving Problem 1 , the spacing errors $e_{i}^{p}(k)$ asymptotically converge to zero and the total platoon is string stable. Now the control Problem 1 is defined.

Problem 1:

For vehicle dynamics (Expression 2) under networked platoon configuration in Figure 1, find the control gain $K_{P}$ and $K_{L}$ in Expression 4 such that the following conditions are satisfied: 
1) Initial condition: for the state error $e_{i}(0) \neq 0$, disturbance variable $\Theta_{i}(k)=0$ and the control input of the leader vehicle $u_{0}(k)=0, \forall i=2,3, \ldots$, n. $\forall k \geq 0$, the bounded state error $e_{i}(k)$ asymptotically converges to zero.

2) For the non-zero and bounded $u_{0}(k)$ and $\Theta_{i}(k)$, the performance output signals (Expressions 11 and 13) satisfy the following constraints, respectively:

$\left\|\xi_{1}\right\|_{2}^{2}<\gamma\left\|u_{0}\right\|_{2}^{2}$

$\left\|\xi_{i}\right\|_{2}^{2}<\mu\left(\left\|R^{1 / 2} e_{i-1}\right\|_{2}^{2}+\vartheta^{-1}\left\|R^{1 / 2} \Theta_{i}\right\|_{2}^{2}\right), i \geq 2$

where the symbol $\|\xi\|_{2}^{2}$ is defined by

$\sum_{k=0}^{\infty} \xi^{T}(k) \xi(k)$.

Remark 3: By Problem 1, $\mathrm{H}_{\infty}$-type performance objectives are handled. The first condition satisfies the asymptotic stability constraint, which can ensure that finite-energy $u_{0}$ will result in finite-energy $e_{i}$ for all $i=2,3 . . . n$, and the desired upper bound on the worst-case energy gain from $u_{0}$ to $\xi_{1}$ is represented by $\gamma$. In Constraint 15, the desired upper bounds can be obtained by $\mu$ and $\mu \vartheta^{-1}$, and the disturbance inputs $e_{i-1}$ and an additional disturbance input $\Theta_{i}$ appears. When $\xi$ is constructed in a way to have $F^{T} F=R$, the error states will at tenuate backward along the platoon if Expression 15 is satisfied by $\mu<1$. The string stable behaviour can be ensured by the value of $\mu$ (e.g. $\mu$ sufficiently small). On the other hand, positive scalar $\vartheta$ is introduced to adjust the desired robustness with communication constraints (with large $\vartheta$ implying better robustness). Our goal is equivalent to deriving the conditions for the solvability of Problem 1 . Here, parameters $\vartheta$ and $R$ have to be fixed beforehand.

Theorem 1: Problem 1 is solvable if there exist $P \in \mathbb{S}_{+}^{3}$ and $T \in \mathbb{R}^{3 \times 1}$ that satisfy

$\left[\begin{array}{ccc}\Psi_{11} & * & * \\ B^{T} & -\gamma \rho^{-1} I & * \\ F_{1} P+G_{1}(H+T) & G_{1} & -\rho l\end{array}\right]<0$

$\left[\begin{array}{cccc}\Psi_{11} & * & * & * \\ H^{T} B^{T} & -\varphi P & 0 & * \\ T^{T} B^{T} & 0 & \varphi \vartheta^{-1} P & * \\ F P+G(H+T) & G H & G T & -l\end{array}\right]<0$

$\left[\begin{array}{cc}\varphi^{-1} P & I \\ l & \mu R\end{array}\right] \geq 0$

where $\Psi_{11}=A P+B(H+T)+P^{T} A^{T}+(H+T)^{T} B^{T}, \rho$ and $\varphi$ are arbitrary positive scalars.

Proof: To obtain the LMI conditions on the solution of Problem 1, we construct the Lyapunov functional as follows

$V(k)=e_{i}^{T}(k) P^{-1} e_{i}$

where $P$ is a strictly positive symmetric matrix. Then, $\Delta V=V(k+1)-V(k)$

$=e_{i}^{T}(k+1) P^{-1} e_{i}(k+1)-e_{i}^{T}(k) P^{-1} e_{i}(k)$

For Expression 15, a sufficient condition is described as
$\Delta V+\xi_{i}^{T}(k) \xi_{i}(k)-\mu e_{i-1}^{T}(k) \operatorname{Re}_{i-1} \mu \vartheta^{-1} \Theta_{i}^{T}(k) R \Theta_{i}<0$ (19)

when $e_{i-1}(k)=0$ and $\Theta_{i}(k)=0$, Inequality 19 can guarantee the stability of the platoon system. For the stable system, $\lim e_{i}(k)=0$ is held. Hence, the sum of Inequality $1 \overrightarrow{9}$ from zero to infinity results in

$$
\begin{aligned}
\left\|\xi_{i}\right\|_{2}^{2}< & \mu\left(\left\|R^{\frac{1}{2}} e_{i-1}\right\|_{2}^{2}+\vartheta^{-1}\left\|R^{\frac{1}{2}} \Theta_{i}\right\|_{2}^{2}\right)+ \\
& +e_{i}^{T}(0) P^{-1} e_{i}(0)
\end{aligned}
$$

when $e_{i}(0)=0$, the Inequality 15 is held. To facilitate the proof, we define:

$\left.\Sigma^{T}(k)\right] \triangleq\left[e_{i}^{T}(k) P^{-1},-e_{i-1}^{T}(k) P^{-1}, \Theta_{i}^{T} P^{-1}, \xi_{i}^{T}(k)\right]$

Then, the Inequality 19 can be written as

$\Sigma^{T}(k)\left[\begin{array}{cccr}\phi_{11} & * & * & * \\ P^{T} K_{P}^{T} B^{T} & -\mu P R P & 0 & * \\ P^{T} K_{L}^{T} B^{T} & 0 & -\mu \vartheta^{-1} P R P * \\ F P+G\left(K_{P} P+K_{L} P\right) & G K_{P} P & G K_{L} P & -I\end{array}\right] \sum(k)<0$

where $\phi_{11}=A P+B\left(K_{P} P+K_{L} P\right)+P^{T} A^{T}+\left(K_{P} P+K_{L} P\right)^{T} B^{T}$. Let $H=K_{P} P$ and $T=K_{L} P$. In order to resolve the nonlinear term $(2,2),(3,3)$ blocks, inspired by the work in [27], we rewrite $(2,2)$ and $(3,3)$ blocks by the following expression

$$
-\mu P R P=\underbrace{(X-\mu P R P)}_{\leq 0}+\underbrace{(-X)}_{<0}
$$

Then, Expression 21 is equivalent to LMI as follows:

$$
\left[\begin{array}{cccc}
\Psi_{11} & * & * & * \\
H^{T} B^{T} & -X & 0 & * \\
T^{T} B^{T} & 0 & -\vartheta^{-1} X & * \\
F P+G(H+T) & G H & G T & -I
\end{array}\right]<0
$$

By the above transformation, no conservatism is introduced as long as $X$ is chosen freely. Hence, let $X=\varphi P$ for getting a set of LMI conditions, where $\varphi$ is an arbitrary positive scalar. By Expression 22, we can obtain $\Sigma^{T}$

$\varphi P^{-1} \leq \mu R$

By applying the Schur complement lemma to Expression 24 and performance objective (Expression 14) for the first vehicle. Theorem 1 holds if LMI Expressions 16, 17 and 18 hold. This completes the proof.

Remark 4: In Problem 1, the $\mathrm{H}_{\infty}$-type performance objectives are formulated with zero initial conditions. However, the nonzero initial conditions are disregarded, which is not practical. For Expression 20, the very large eigenvalues of $P^{-1}$ might bring about some severe consequences. However, it should be pointed out that Expression 24 limits the choice of $P^{-1}$. So, we can remove the adverse effects of nonzero initial conditions by the choices of sufficiently small values for $\varphi$ and $\rho / \varphi^{-1}$. Similarly, it can also be applied to the first vehicle.

Remark 5: In this paper, the imperfect communication is merged into multiple input delay problems. The upper bounded of network-induced delays $\tau_{i}^{k}$ can influence the feasibility of Equations 16-18. note that the platoon system is unstable when the delay $\tau_{i}^{k}$ is greater than a certain value. In this sense, the asymptotic and string stability of the platoon 
system can be affected by the upper bound of network-induced delays $\tau_{i}^{k}$.

\section{SIMULATIONS AND EXPERIMENTS}

\subsection{Platoon control system with multiple time delays}

This section shows the efficiency of the developed method via numerical simulations. Four vehicles running on a horizontal road are considered. Set the sampling interval $T=0.005$, the engine time constant $\eta=0.2$, the length of vehicle $L=5 \mathrm{~m}$ and the desired vehicle spacing $e_{d}^{p}=12 \mathrm{~m}$. Here, we set the parameter $s=0.1, \gamma=0.9, \rho=\varphi=1.2$. It is assumed that the initial velocities of the vehicles are. Without losing generality, the acceleration for the leader vehicle is described as

$a_{0}= \begin{cases}2 m / s^{2} & 10 \leq t \leq 20 \\ -1 m / s^{2} & 50 \leq t \leq 60 \\ 0 & \text { others }\end{cases}$

It is easy to check by using the Matlab LMI Toolbox that Expressions 16, 17 and 18 are feasible and we obtain the controller gain $K_{P}$ and $K_{L}$ below

$K_{P}=[-4.8170,-3.0746,-0.1768]$,

$K_{L}=[-12.5143,-3.4666,-1.7546]$

As the leader vehicle is the directly preceding vehicle for the first vehicle in the platoon, the first vehicle works without the communication effect. In order to show it clearly, the first 50 of 5,000 random distributed time-varying delays $\left(0 \leq \tau_{i}^{k} \leq 5\right)$ are shown in Figure 4 , where the communication effect of the platoon is transformed into time-varying delays by SDP, no matter what communication delay, packet dropout and disorder, as shown in Section 2. The tracking distance error responses of five following vehicles are shown in Figure 5. Figure 5 indicates that the controller can stabilize the system with time delays and maintain the desired inter-vehicle distance $\left(e_{1}, e_{2}, e_{3}\right)$. Figures 6,7 and 8 show velocity, position and acceleration of the following vehicles, respectively. From Figures 6,7 and 8 it can be clearly seen that the following vehicles have good tracking performance for the leader vehicle accelerating or slowing down.

In the same case, when the method proposed in [24] is used, the spacing distance error between two adjacent vehicles and the velocity of the vehicles are shown in Figures 9 and 10. By comparing Figure 5 with Figure 9 , it is shown that the spacing distance error $e_{i}$ backwards gradually reduces to accomplish the string stability which is given in [24]. Although the control law in [24] can obtain a smaller distance error than this paper, the control law in this paper makes the spacing distance error converge to zero without overshoot, fast speed and stationary. It can effectively improve the safety and comfort of vehicles. From this view, the controller of platoon in this paper is better than in [24].

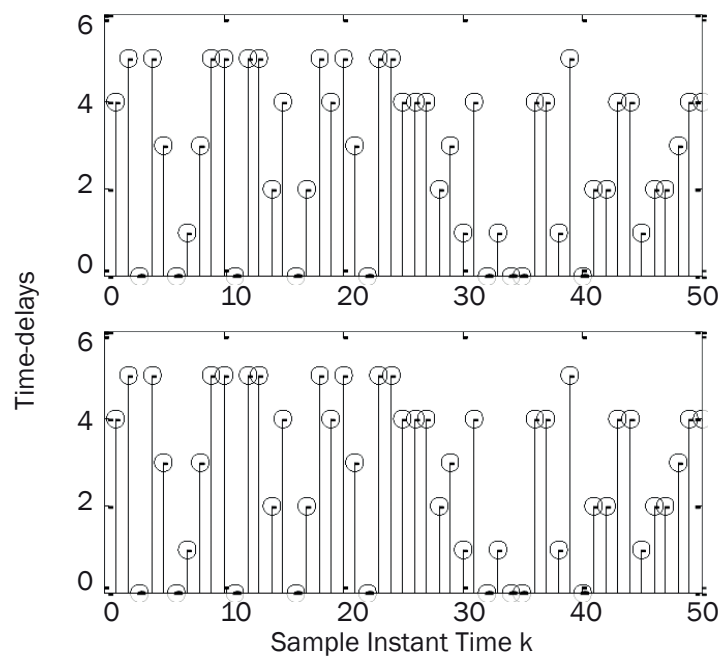

Figure 4 - Random distributed time-varying delays of inter-vehicle communication

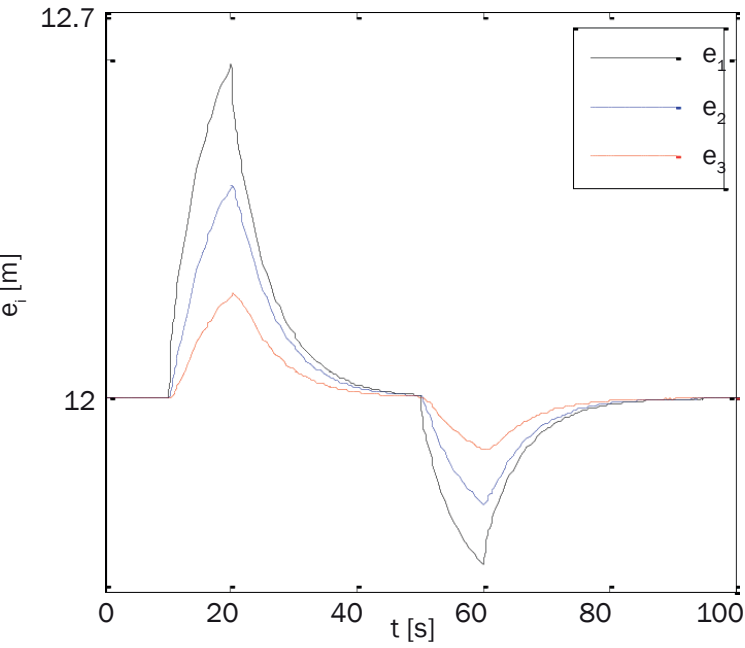

Figure 5 - The spacing distance error between two adjacent vehicles

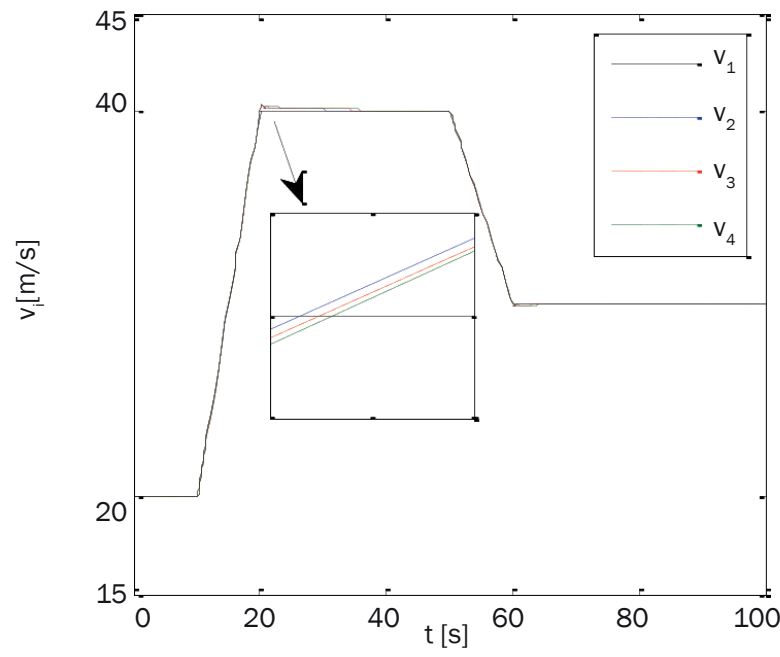

Figure 6 - The velocity of the 4 vehicles 


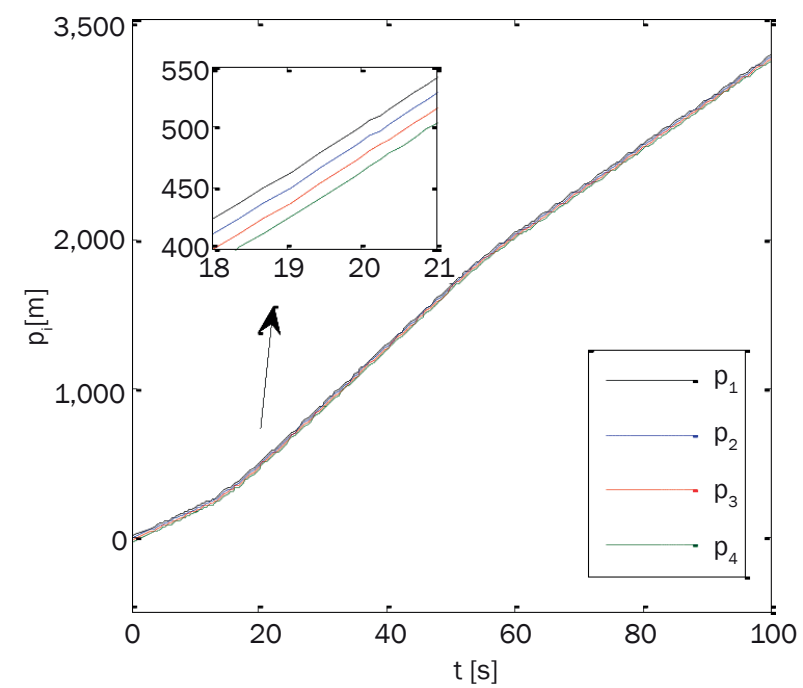

Figure 7 - The position of the 4 vehicles

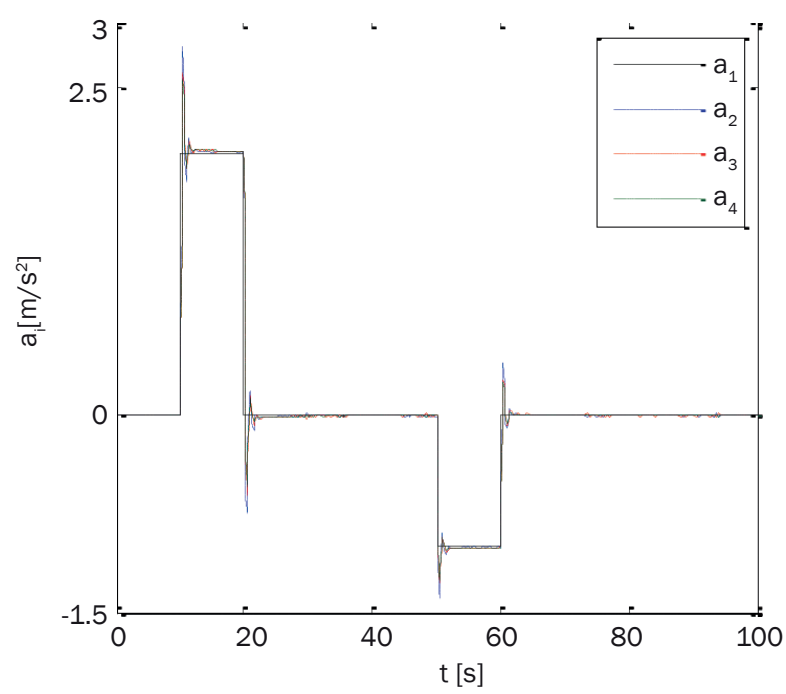

Figure 8 - The acceleration of the 4 vehicles

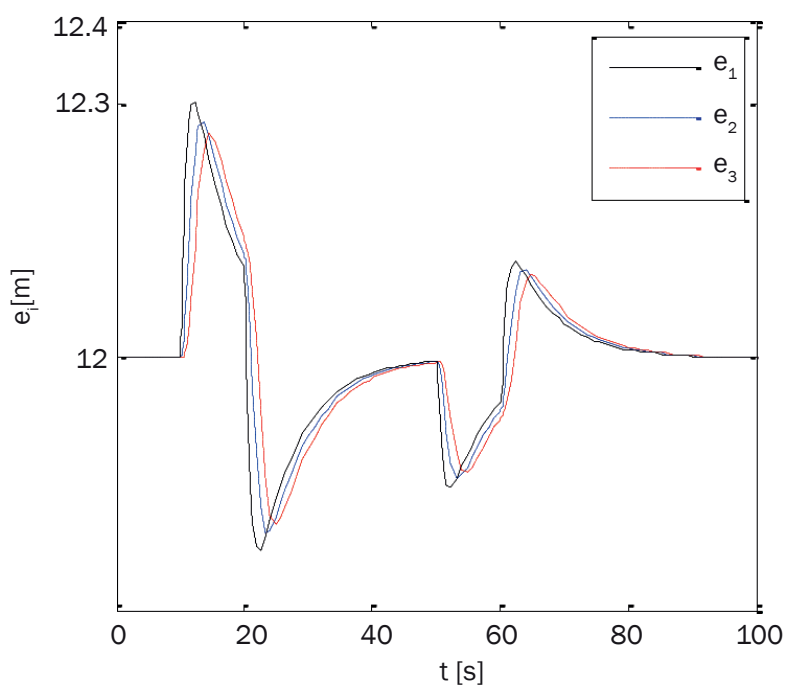

Figure 9 - Spacing distance error between two adjacent vehicles [24]

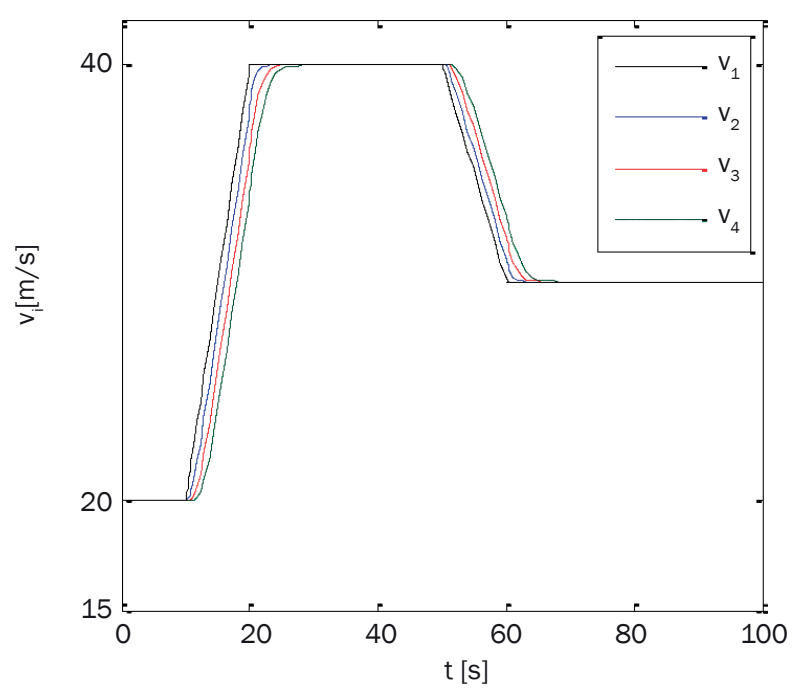

Figure 10 - The velocity of the vehicles [24]

\subsection{Experiments with Arduino cars}

The experiments show that the proposed smart data processing mechanism and the multi-objective $\mathrm{H}_{\infty}$-type synthesis are effective in practice by using four Arduino cars (in Figures 11 and 12). The Arduino car is driven and steered by two front wheels. The longitudinal speed and acceleration of the Arduino car are detected by an incremental encoder sensor installed on the shaft of the rear wheels and an acceleration sensor installed on the top of the Arduino car. The cameras mainly keep each car running a straight line. For each car, an on-board Arduino processor serves as the real-time computing and control unit.

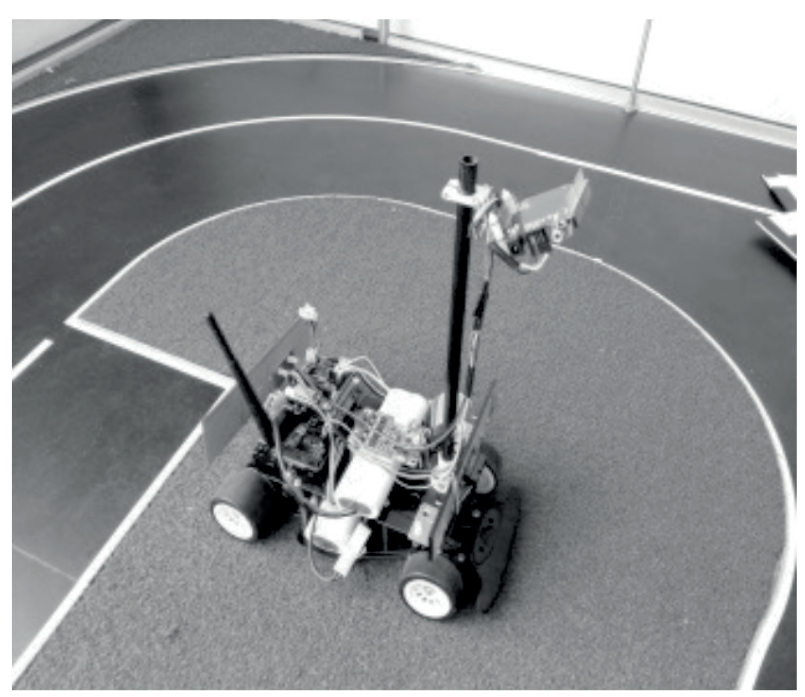

Figure 11 - Arduino car 


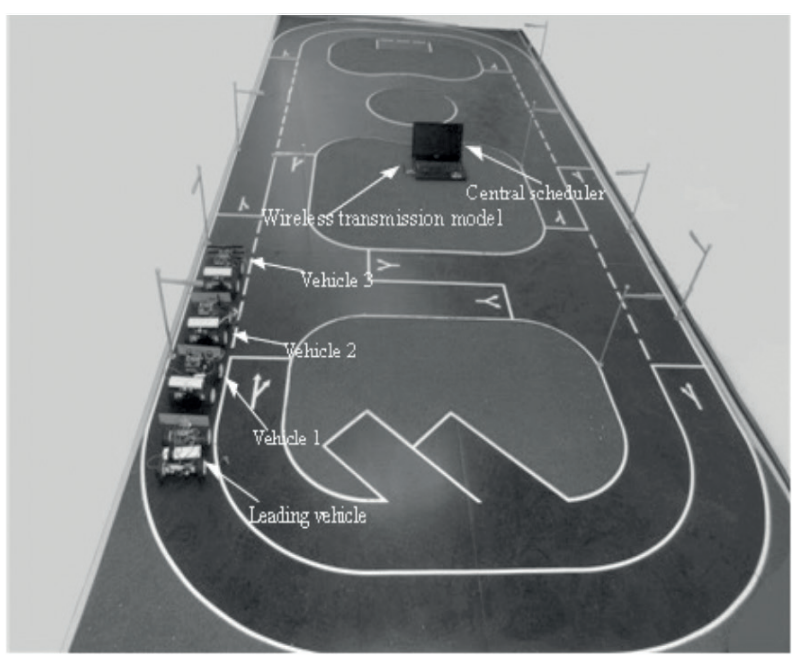

Figure 12 - Four Arduino cars in platoon

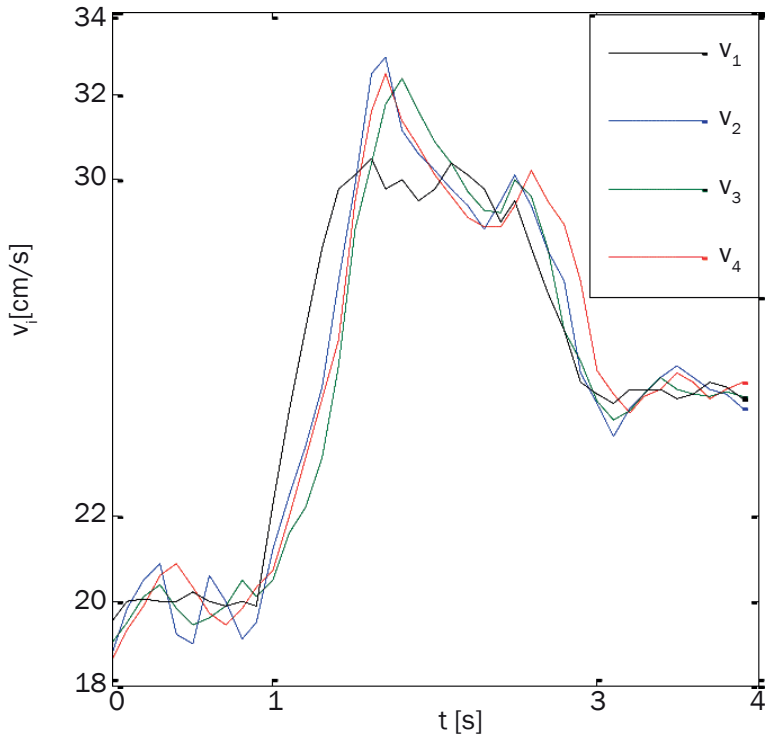

Figure 13 - The spacing distance errors

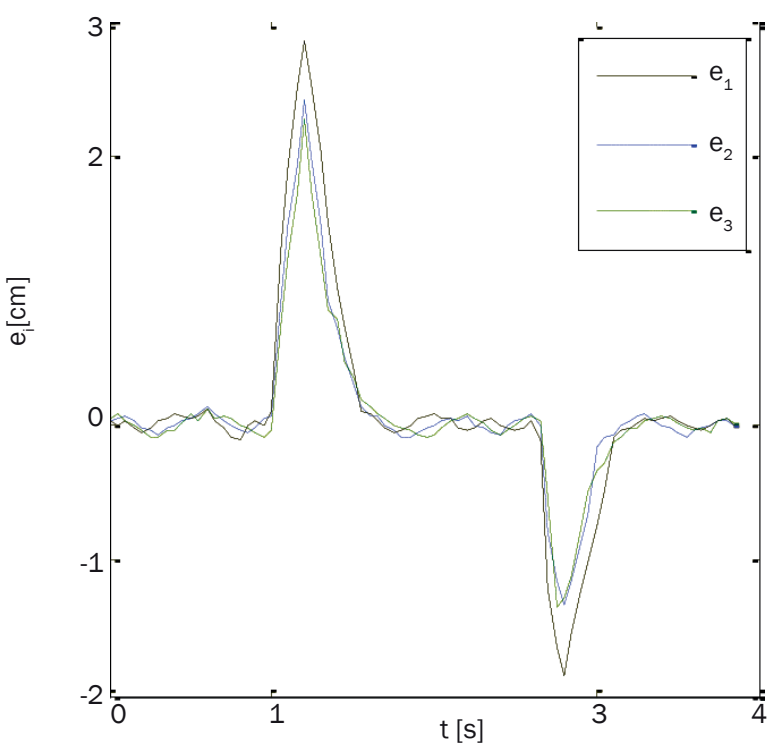

Figure 14 - The velocity of the following Arduino cars response
Since Arduino cars need to be very close to each other (e.g. the spacing distance errors can be as small as several centimetres), the following Arduino cars obtain the state information of its directly preceding car by on-board sensor and of the leader car by wireless communication. Here, the wireless module uses APC220, whose working frequency is $418 \mathrm{MHz}$ to 455 $\mathrm{MHz}$ and the transmission distance is 1,000 metres. The single-packet transmission policy is used in the experiments. As the leader car 0 is the directly preceding car for car 1 in the platoon, car 1 run without the communication effect. Cars 2 and 3 receive the state data packets of the leader car 0 , so the communication delay, packet dropout and disorder may occur during the data packets transmission.

In the experiments, the leader car 0 is assumed to run on a horizontal road at $20 \mathrm{~cm} / \mathrm{s}$, and the leader car 0 speeds up at the time instant 2 and slows down at the time instant 5 . The desired spacing between two consecutive cars is given by $e_{i}^{p}(k)=5 \mathrm{~cm}$. The time-varying delays $\tau_{i}^{k}$ are depicted in Figure 4 . Here, the time-varying delays are achieved via software programming. The spacing distance errors of the two consecutive cars are shown in Figure 13 and the velocities of the Arduino cars are shown in Figure 14. In Figures 13 and 14, it is clearly seen that all following Arduino cars are asymptotically stable and the platoon string stability can be guaranteed. In the experiments, it is worth noting that the following cars do not obtain good tracking performance when delays $\tau_{i}^{k}$ are set to be more than $0.5 \mathrm{~s}$.

\section{CONCLUSION}

This paper investigates the platoon control system with communication delays, disorders and packet losses. An SDP is proposed to deal with the time-varying delays, dropout disorders and asynchronous data packets in vehicular cooperative control. Based on SDP mechanism, the platoon control problem is transformed into a multi-objective $\mathrm{H}_{\infty}$-type synthesis problem, which can achieve robustness against communication constraints. A new platoon model based on network communication has been presented. Based on the model, a controller, which guarantees that the platoon control system is asymptotically stable and string stable, is designed. Numerous simulations and experiments with laboratory scale Arduino cars results show the efficiency of the proposed methods.

In future research, we will plan to study the time delays and packet loss with limited conditions. A networked-predictive-control scheme is employed to compensate the effect of communication constraints.

\section{ACKNOWLEDGEMENT}

This study has been supported by the National Natural Science Foundation of China (Grant No. 61503003) 
and the Postdoctoral Science Foundation of China (No. 2015M581332) and the Natural Science Foundation of Anhui Province (Grant No. 1508085QF126).

吴利刚，博士，讲师，单位：中国山西大同大学煤 炭工程学院

卢自宝，博士，讲师，单位：中国大连理工大学控 制科学与工程学院

郭戈, 博士, 教授, 单位: 中国大连海事大学信息 科学技术学院

通信受限的网络化自主车队分析, 综合与实验研究

\section{摘要}

本文研究了网络化自主车队中存在的通信时延、 丢包和数据包乱序问题。通过设计每辆车的智能数 据处理器 (SDP) 来解决通信受限问题。基于该处理 机制, 提出了领头车-前车跟随策略。将具有通信时 延的车队控制问题转化为多目标的 $H \infty$ 综合问题，保 证了车队的渐近稳定和队列稳定性。最后通过求解 线性矩阵不等式, 给出了满足上述稳定性控制器增 益的充分条件。数值仿真和Arduino智能小车实验验 证了本文提出算法的正确性和实用性。

\section{关键词}

自主车队, 通信受限, 智能数据处理器, $H \infty$ 性能

\section{REFERENCES}

[1] Huang S, Ren W. Safety, comfort, and optimal tracking control in AHS applications. IEEE Control Systems Magazine. 1998;18(4):50-64.

[2] Placzek B. Selective data collection in vehicular networks for traffic control applications. Transport Res. C-Emer. 2012;23(2):14-28.

[3] Toor Y, Muhlethaler P, Laouiti A. Vehicle ad hoc networks: applications and related technical issues. IEEE Commun. Surv. Tut. 2008;10(3):74-88.

[4] Varaiya P. Smart cars on smart roads: Problem of control. IEEE Transactions on Automatic Control. 1993;38(2):195-207.

[5] Barooah P, Mehta P. G., Hespanha J. P.. Mistun ing-based control design to improve closed-loop stability margin of vehicular platoons. IEEE Transactions on Automatic Control. 2009;54(9):2100-2113.

[6] Dunbar W, Caveney D. Distributed receding horizon control of vehicle platoons: Stability and string stability. IEEE Transactions on Automatic Control. 2012;57(3):620-633.

[7] Kianfar R, Augusto B, Ebadighajari A. Design and experimental validation of a cooperative driving system in the grand cooperative driving challenge. IEEE Transactions on Intelligent Transportation Systems. 2012;13(3):99-1007.

[8] Seiler P, Pant A, Hedrick K. Disturbance propagation in vehicle strings. IEEE Transactions on Automatic Control. 2004;49(10):1835-1841.

[9] Swaroop D, Hedrick JK, Choi SB. Direct adaptive longitudinal control of vehicle platoons. IEEE Transactions on Vehicular Technology. 2001;50(1):150-161.

[10] Caveney D. Cooperative vehicular safety applications. IEEE Control Systems Magazine. 2010;30(4):38-53.
[11] Guo G, Wang LY. Control over Medium-Constrained Vehicular Networks with Fading Channels and Random Access Protocol: A Networked Systems Approach. IEEE Transactions on Vehicular Technology. 2014;99(5):289-296.

[12] Arem B, Driel CJG, Visser R. The impact of cooperative adaptive cruise control on traffic-flow characteristics. IEEE Transactions on Intelligent Transportation Systems. 2006;7(4):429-436.

[13] Zheng Y, et al. Influence of information flow topology on closed-loop stability of vehicle platoon with rigid formation. Intelligent Transportation Systems (ITSC), 2014 IEEE 17th International Conference on. IEEE, 2014.

[14] Zheng Y, Li SE, et. al. Stability and Scalability of Homogeneous Vehicular Platoon: Study on Influence of Information Flow Topologies. IEEE Transactions on Intelligent Transportation Systems. 2016;17(1):14-26.

[15] Zheng Y, Li YS, Li KQ, Wang LY. Stability Margin Improvement of Vehicular Platoon Considering Undirected Topology and Asymmetric Control. IEEE Transactions on Control Systems Technology. 2016;24(4):1253-1265.

[16] Liu X, Goldsmith A, Mahal SS, Hedrick JK. Effects of communication delay on string stability in vehicle platoons. Proc. IEEE Intl. Conf. Intell. Transp. Syst. 2001;99(3):625-630.

[17] ncu S, Van de Wouw N, Nijmeijer H. String stability of interconnected vehicles under communication constraints. Proc. IEEE Conf. Decision Control. 2012;24592464.

[18] Xiao LY, Gao F. Effect of information delay on string stability of platoon of automated vehicles under typical information frameworks. Journal of Central South University of Technology. 2010;17(6):1271-1278.

[19] Guo G, Yue W. Autonomous Platoon Control Allowing Range-Limited Sensors. IEEE Transactions on Vehicular Technology. 2012;61(7):2901-2912.

[20] Yue W, Guo G. Guaranteed cost adaptive control of nonlinear platoons with actuator delay. ASME Journal of Dynamic System Measurement and Control. 2012;134(5):1-11.

[21] Ghasemi A, Kazemi R, Azadi S. Stability analysis of a predecessor-following platoon of vehicles with two time delays. PROMET-Traffic \&Transportation. 2015;27(1):35-46.

[22] Hakan K, Paolo F. State feedback synthesis for homogenous platoons under the leader and predecessor following scheme. European Control Conference (ECC); 2014 June 24-27; Strasbourg, France; 2014.

[23] Öncu S, Ploeg J, Van de Wouw N, Nijmeijer H. Cooperative Adaptive Cruise Control: Network-Aware Analysis of String Stability. IEEE Transactions on Intelligent Transportation Systems. 2014;15(4):225-234.

[24] Guo G, Yue W. Hierarchical platoon control with heterogeneous information feedback. IET Control Theory Appl. 2011;5(15):1766-1781.

[25] Xiong J, Lam J. Stabilization of networked control systems with a logic ZOH. IEEE Transactions on Automatic Control. 2009;54(2):358-363.

[26] Zhang X, Han Q. Network-based filtering for discrete-time systems. IEEE Transactions on Signal Processing. 2012;60(2):956-961.

[27] Köroğlu H. Observer-based LPV control with guaranteed -gain and -type performance objectives. International Journal of Robust and Nonlinear Control. 2014;24(14):2000-2017. 